\title{
Comparative genomic reconstruction of transcriptional networks controlling central metabolism in the Shewanella genus
}

Dmitry A Rodionov ${ }^{1,2^{*}+}$, Pavel S Novichkov ${ }^{3+}$, Elena D Stavrovskaya ${ }^{2,4}$, Irina A Rodionova ${ }^{1}$, Xiaoqing Li ${ }^{1}$, Marat D Kazanov ${ }^{1,2}$, Dmitry A Ravcheev ${ }^{1,2}$, Anna V Gerasimova ${ }^{3}$, Alexey E Kazakov², Galina Yu Kovaleva², Elizabeth A Permina ${ }^{5}$, Olga N Laikova ${ }^{5}$, Ross Overbeek ${ }^{6}$, Margaret F Romine ${ }^{7}$, James K Fredrickson ${ }^{7}$, Adam P Arkin $^{3}$, Inna Dubchak ${ }^{3,8}$, Andrei L Osterman ${ }^{1,6}$, Mikhail S Gelfand ${ }^{2,4}$

\begin{abstract}
Background: Genome-scale prediction of gene regulation and reconstruction of transcriptional regulatory networks in bacteria is one of the critical tasks of modern genomics. The Shewanella genus is comprised of metabolically versatile gamma-proteobacteria, whose lifestyles and natural environments are substantially different from Escherichia coli and other model bacterial species. The comparative genomics approaches and computational identification of regulatory sites are useful for the in silico reconstruction of transcriptional regulatory networks in bacteria.

Results: To explore conservation and variations in the Shewanella transcriptional networks we analyzed the repertoire of transcription factors and performed genomics-based reconstruction and comparative analysis of regulons in 16 Shewanella genomes. The inferred regulatory network includes 82 transcription factors and their DNA binding sites, 8 riboswitches and 6 translational attenuators. Forty five regulons were newly inferred from the genome context analysis, whereas others were propagated from previously characterized regulons in the Enterobacteria and Pseudomonas spp.. Multiple variations in regulatory strategies between the Shewanella spp. and E. coli include regulon contraction and expansion (as in the case of PdhR, HexR, $\mathrm{FadR}$ ), numerous cases of recruiting non-orthologous regulators to control equivalent pathways (e.g. PsrA for fatty acid degradation) and, conversely, orthologous regulators to control distinct pathways (e.g. TyrR, ArgR, Crp).

Conclusions: We tentatively defined the first reference collection of $\sim 100$ transcriptional regulons in 16 Shewanella genomes. The resulting regulatory network contains $\sim 600$ regulated genes per genome that are mostly involved in metabolism of carbohydrates, amino acids, fatty acids, vitamins, metals, and stress responses. Several reconstructed regulons including NagR for $\mathrm{N}$-acetylglucosamine catabolism were experimentally validated in S. oneidensis MR-1. Analysis of correlations in gene expression patterns helps to interpret the reconstructed regulatory network. The inferred regulatory interactions will provide an additional regulatory constrains for an integrated model of metabolism and regulation in S. oneidensis MR-1.
\end{abstract}

\footnotetext{
* Correspondence: rodionov@burnham.org

† Contributed equally

${ }^{1}$ Sanford-Burnham Medical Research Institute, La Jolla, California, USA

Full list of author information is available at the end of the article
} 


\section{Background}

Fine-tuned regulation of gene expression in response to extracellular and intracellular signals is a key mechanism for successful adaptation of microorganisms to changing environmental conditions. Activation and repression of gene expression in bacteria is usually mediated by DNA-binding transcription factors (TFs) that specifically recognize TF-binding sites (TFBSs) in upstream regions of target genes, and also by various regulatory RNA structures including cis-acting metabolite-sensing riboswitches and attenuators encoded in the leader regions of target genes. Genes and operons directly co-regulated by the same TF or by an RNA structure are considered to belong to a regulon. All regulons taken together form the transcriptional regulatory network (TRN) of the cell. TFs form more than 50 different protein families and constitute around $5-10 \%$ of all genes in an average bacterial genome, and their respective regulons cover a substantial fraction of bacterial TRNs [1].

Traditional experimental methods for the analysis of transcriptional gene regulation and characterization of TFBSs provided a foundation for the current understanding of regulatory interactions [2]. However, taken alone, they are limited in productivity (the scale) and feasibility (often restricted to a few model organisms). High-throughput transcriptome approaches opens new opportunities for measuring the expression of thousands of genes in a single experiment [3]. The microarray technology has been successfully used to explore transcriptional responses in several bacteria. However, convoluted regulatory cascades, multi-TF regulation of certain genes, and various indirect effects on the transcription and abundance of mRNA make the observed regulatory responses too complex for a direct top-down analysis. The chromatic immunoprecipitation approach is now increasingly used for the investigation of genome-wide DNA-binding of global TFs in bacteria [3]. At the same time, a growing number of complete prokaryotic genomes allows us to extensively use comparative genomics approaches to infer conserved cis-acting regulatory elements (e.g. TFBSs and riboswitches) in regulatory networks of numerous groups of bacteria ([4-15], also reviewed in [1]). These and other previous studies enabled us to define and prototype a general workflow of the "knowledge-driven" approach for the comparative-genomic reconstruction of regulons. Two major components of this analysis are (i) propagation of previously known regulons from model organisms to others and (ii) ab initio prediction of novel regulons (see Methods for more details). This approach is different, and in many ways complementary to the two most common alternative approaches to the TRN reconstruction: (i) the "data-driven" approach, top-down regulatory network reconstruction from microarray data [16]; and (ii) the "computation-driven" approach, ab initio automated identification and clustering of conserved DNA motifs [17] .

Shewanella spp. are Gram-negative facultative anaerobic $\gamma$-proteobacteria characterized by a remarkable versatility in using a variety of terminal electron acceptors for anaerobic respiration (reviewed in [18]). Isolated from various aquatic and sedimentary environments worldwide, the Shewanella demonstrate diverse metabolic capabilities and adaptation for survival in extreme conditions (Fig. 1) [19]. Although the model species Shewanella oneidensis MR-1 is a subject of extensive genetics and physiological studies, as well as genome-scale transcriptomics and proteomics approaches [18,20-22], our experimental knowledge of transcriptional regulation in S. oneidensis is limited to the Fur, ArcA, TorR, Crp, and EtrA (Fnr) TFs controlling iron metabolism and anaerobic respiration [23-29]. In addition, the novel NrtR regulon for NAD cofactor metabolism was inferred by comparative genomics and experimentally validated in S. oneidensis[11].

Availability of multiple closely-related genomes from the Shewanella genus (Fig. 1) provided a basis for the reconstruction of the metabolic and regulatory networks using comparative genomics. Recently, we have applied the comparative genomic approach to predict novel pathways and regulons for the $N$-acetylglucosamine and lactate utilization [30,31], and to reconstruct two novel regulons for the fatty acid and branched-chain amino acid utilization pathways in Shewanella spp. [4]. In this study, we have extended our previous analysis towards the detailed reconstruction of $\sim 100$ transcriptional regulons in 16 Shewanella species with completely sequenced genomes. The identified TRN contains over 450 regulated genes per genome, mostly covering the central and secondary metabolism and stress response pathways. The comparative analysis of the reconstructed regulons revealed many aspects of the metabolic regulation in the Shewanella that are substantially different from the established TRN model of Escherichia coli.

\section{Results}

\section{Repertoire of transcription factors in the Shewanella spp}

Previous comparative analysis revealed extensive gene content diversity among 10 Shewanella genomes [32]. To gain further insight into the scale of the TRN diversity in this lineage, we analyzed the repertoire of DNAbinding TFs encoded in 16 complete Shewanella genomes (Additional file 1). The total number of TFs in individual species varies broadly, from 138 TFs in $S$. denitrificans to 262 TFs in $S$. woodyi, with an average of 200 TFs per genome (Fig. 2). 95\% of all TFs of the 
A.

Shewanella amazonensis SB2B [Amazon river delta, Brazil; sediment, suboxic redox conditions; $1 \mathrm{~m}$ ]

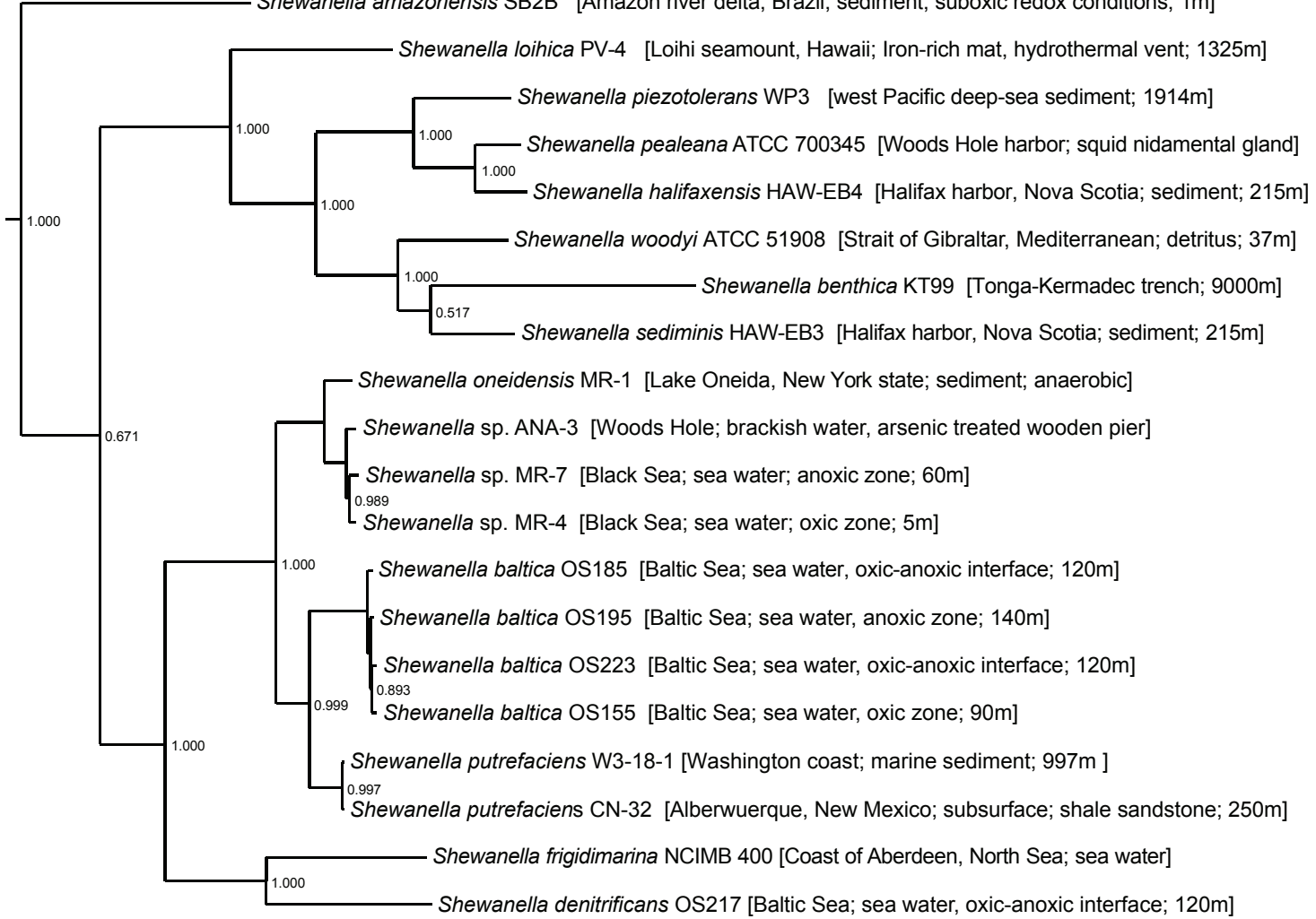

B.

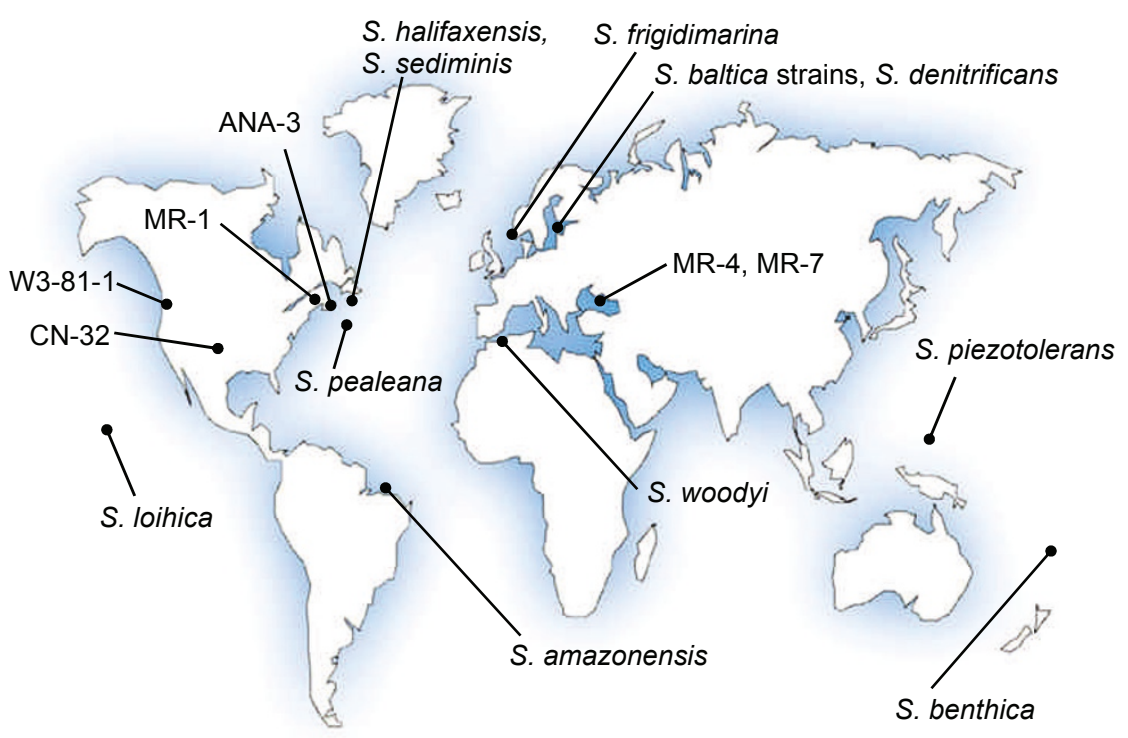

Figure 1 Phylogenetic tree, isolation site characteristics and the geographic origin of 20 Shewanella species with available genomes. The tree was constructed using the concatenated alignment of $\sim 78$ universal prokaryotic proteins in the MicrobesOnline database http://www. microbesonline.org/cgi-bin/speciesTree.cgi. 
Shewanella belong to 17 major protein families with at least two distinct members per genome. At that, the total number of TFs in most of these families varies significantly among the Shewanella spp. The largest TF families are LysR, OmpR, Fis, TetR, AraC, and LuxR (>10 TFs per genome on average). Among the remaining 14 families of TFs, mostly represented by single members in the genomes (without paralogs), the Fur, ArgR, BirA, LexA, MetJ, NrdR, RpiR, and TrpR families are universally conserved in the Shewanella (Fig. 2). A significant reduction of the TF repertoire is a unique feature of $S$. denitrificans, which has limited anaerobic growth capabilities due to massive gene loss in course of ecological specialization [32].

The 3,228 predicted TFs in 16 Shewanella genomes were clustered into 686 orthologous groups (Additional file 1), among which only 63 TFs (9\%) were universally conserved in all genomes (the core TF set), 320 TFs $(47 \%)$ were found in at least two genomes (variable TFs), whereas the remaining TFs (303 or 44\%) were strain-specific (Additional file 1). Although the genomes of the Shewanella spp. and E. coli demonstrate a similar repertoire and size of TF protein families, only 73 (30\%) TFs from E. coli have orthologs in at least one Shewanella genome (Fig. 2). The group of 34 TFs that are present in the Shewanella core TF set (Additional file 2) and conserved between E. coli and the Shewanella spp. (Additional file 3) is enriched by regulators controlling the metabolism of amino acids (ArgR, AsnC, CysB, GcvA, IlvY, MetJ, MetR, TrpR, TyrR), fatty acids (FabR,
FadR), cofactors (BirA, IscR), deoxynucleosides (NrdR), nitrogen (NtrC), phosphate (PhoB), iron (Fur), central carbohydrate metabolism (HexR, PdhR), stress responses (CpxR, LexA, NhaR, NsrR), and global regulators (ArcA, Crp, Fis, Fnr, and Lrp). The group of strain-specific Shewanella regulators with orthologs in E. coli contains 5 known regulators for local carbohydrate utilization pathways (AlgR, NanR, DgoR, GalR, GntR) that were possibly acquired together with the target metabolic pathway genes via lateral gene transfer events [33]. Near $1 / 2$ of strain-specific TFs of the Shewanella spp. belong to two protein families, LysR and AraC (96 and 50 TFs, respectively), that were likely expanded via gene duplication in course of ecological adaptation of individual species.

\section{Comparative analysis of transcriptional regulation in the Shewanella spp}

To infer TRNs in the Shewanella spp., we used the integrative comparative genomics approach that combines identification of TFs and candidate TFBSs with crossgenomic comparison of regulons and with the genomic and functional context analysis of candidate target genes. We analyzed 16 Shewanella genomes and inferred regulons for 82 orthologous groups of TFs that split into two groups: 41 regulators with experimentally characterized orthologs in S. oneidensis or other $\gamma$-proteobacteria (Table 1), and 41 novel regulators without characterized orthologs in any species (Table 2). The genomic and functional content of the reconstructed TF regulons from both groups, as well as of the regulons controlled by

\begin{tabular}{|c|c|c|c|c|c|c|c|c|c|c|c|c|c|c|c|c|c|c|c|c|c|c|c|c|c|c|c|c|c|c|}
\hline Genome & $\begin{array}{l}0 \\
\\
\end{array}$ & $\frac{\alpha}{\infty}$ & 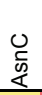 & $\begin{array}{l}0 \\
0 \\
0 \\
0\end{array}$ & $\frac{0}{\underline{v}}$ & $\begin{array}{l}\frac{r}{0} \\
0 \\
0 \\
\end{array}$ & $\stackrel{\frac{\infty}{1}}{1}$ & 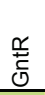 & 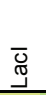 & $\stackrel{\frac{\alpha}{x}}{3}$ & $\stackrel{\frac{n}{0}}{3}$ & 觉 & 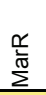 & $\frac{\substack{\frac{\alpha}{0} \\
\sum}}{\sum}$ & 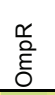 & 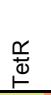 & 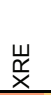 & $\begin{array}{l}\mathbb{N} \\
\underline{\underline{I}} \\
\end{array}$ & 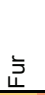 & 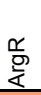 & 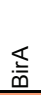 & 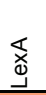 & $\stackrel{+}{ \pm 0}$ & $\begin{array}{l}\frac{n}{0} \\
\frac{0}{z}\end{array}$ & $\frac{\underline{\alpha}}{\frac{\alpha}{\alpha}}$ & $\begin{array}{l}\frac{\alpha}{2} \\
\text { 는 }\end{array}$ & $\begin{array}{l}\frac{r}{\pi} \\
\frac{\pi}{0} \\
\infty\end{array}$ & 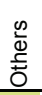 & $\begin{array}{c}\text { Total } \\
\text { TFs } \\
\end{array}$ & $\begin{array}{c}\text { TF } \\
\text { fracti- } \\
\text { on, } \% \\
\end{array}$ \\
\hline E.coli K12 & 29 & 2 & 3 & 0 & 2 & 13 & 15 & 20 & 14 & 24 & 46 & 1 & 3 & 5 & 17 & 13 & 1 & 2 & 2 & 1 & 1 & 1 & 1 & 1 & 4 & 1 & 1 & 14 & 237 & 5.5 \\
\hline S.oneidensis MR-1 & 9 & 4 & 5 & 1 & 4 & 2 & 14 & 7 & 2 & 11 & 53 & 5 & 6 & 5 & 24 & 17 & 10 & 2 & 1 & 1 & 1 & 1 & 1 & 1 & 1 & 1 & 1 & 3 & 193 & 4.5 \\
\hline S. putrefaciens $\mathrm{CN}-32$ & 9 & 6 & 4 & 1 & 4 & 2 & 18 & 12 & 1 & 9 & 44 & 4 & 8 & 7 & 22 & 14 & 8 & 2 & 1 & 1 & 1 & 1 & 1 & 1 & 1 & 1 & 1 & 5 & 189 & 4.8 \\
\hline S.putrefaciens W3-18-1 & 10 & 7 & 4 & 1 & 4 & 2 & 17 & 12 & 1 & 9 & 41 & 4 & 8 & 7 & 21 & 15 & 9 & 2 & 1 & 1 & 1 & 1 & 1 & 1 & 1 & 1 & 1 & 4 & 187 & 4.6 \\
\hline Shewanella sp. ANA-3 & 17 & 6 & 4 & 2 & 3 & 3 & 18 & 10 & 3 & 9 & 49 & 5 & 10 & 9 & 30 & 18 & 11 & 2 & 1 & 1 & 1 & 1 & 1 & 1 & 1 & 1 & 1 & 2 & 220 & 5.4 \\
\hline Shewanella sp. MR-4 & 13 & 3 & 4 & 1 & 2 & 3 & 16 & 8 & 3 & 9 & 44 & 6 & 10 & 7 & 30 & 17 & 5 & 2 & 1 & 1 & 1 & 1 & 1 & 1 & 1 & 1 & 1 & 1 & 193 & 4.9 \\
\hline Shewanella sp. MR-7 & 12 & 3 & 4 & 1 & 2 & 3 & 15 & 10 & 4 & 8 & 44 & 6 & 10 & 6 & 30 & 16 & 6 & 2 & 1 & 1 & 1 & 1 & 1 & 1 & 1 & 1 & 1 & 1 & 192 & 4.8 \\
\hline S. baltica OS155 & 16 & 5 & 5 & 0 & 3 & 2 & 15 & 12 & 5 & 9 & 51 & 5 & 9 & 6 & 21 & 16 & 13 & 2 & 1 & 1 & 1 & 1 & 1 & 1 & 1 & 1 & 1 & 2 & 206 & 4.8 \\
\hline S. denitrificans OS217 & 7 & 2 & 3 & 1 & 3 & 1 & 18 & 6 & 3 & 5 & 24 & 4 & 7 & 6 & 15 & 15 & 7 & 2 & 1 & 1 & 1 & 1 & 1 & 1 & 1 & 1 & 0 & 1 & 138 & 3.7 \\
\hline S. frigidimarina NCIMB 400 & 6 & 5 & 4 & 0 & 2 & 2 & 20 & 8 & 4 & 9 & 50 & 2 & 10 & 8 & 24 & 20 & 9 & 2 & 2 & 1 & 1 & 1 & 1 & 1 & 1 & 1 & 1 & 0 & 195 & 4.8 \\
\hline S.amazonensis SB2B & 8 & 3 & 6 & 1 & 3 & 4 & 15 & 7 & 5 & 9 & 40 & 4 & 6 & 6 & 30 & 16 & 2 & 2 & 1 & 1 & 1 & 1 & 1 & 1 & 1 & 1 & 0 & 1 & 176 & 4.8 \\
\hline S. loihica PV-4 & 14 & 5 & 6 & 1 & 3 & 2 & 17 & 8 & 2 & 11 & 49 & 4 & 9 & 5 & 25 & 18 & 8 & 2 & 1 & 1 & 1 & 1 & 1 & 1 & 1 & 1 & 1 & 1 & 199 & 5.2 \\
\hline S. pealeana ATCC 700345 & 15 & 4 & 6 & 4 & 3 & 1 & 18 & 10 & 3 & 11 & 64 & 3 & 6 & 4 & 20 & 19 & 9 & 2 & 1 & 1 & 1 & 1 & 1 & 1 & 1 & 1 & 1 & 2 & 213 & 5.0 \\
\hline S. halifaxensis HAW-EB4 & 15 & 4 & 6 & 4 & 3 & 1 & 17 & 8 & 3 & 15 & 66 & 3 & 4 & 5 & 19 & 20 & 7 & 2 & 1 & 1 & 1 & 1 & 1 & 1 & 1 & 1 & 1 & 1 & 212 & 5.0 \\
\hline S. piezotolerans WP3 & 19 & 8 & 6 & 2 & 4 & 1 & 19 & 8 & 3 & 11 & 61 & 2 & 7 & 5 & 19 & 21 & 7 & 2 & 1 & 1 & 1 & 1 & 1 & 1 & 1 & 1 & 1 & 1 & 215 & 4.2 \\
\hline S. sediminis HAW-EB3 & 14 & 6 & 5 & 2 & 5 & 2 & 21 & 6 & 1 & 18 & 74 & 3 & 9 & 5 & 24 & 22 & 9 & 2 & 1 & 1 & 1 & 1 & 1 & 1 & 1 & 1 & 1 & 1 & 238 & 5.3 \\
\hline S. woodyi ATCCC 51908 & 29 & 5 & 5 & 4 & 3 & 2 & 22 & 12 & 5 & 16 & 64 & 6 & 12 & 6 & 27 & 18 & 14 & 2 & 1 & 1 & 1 & 1 & 1 & 1 & 1 & 1 & 1 & 1 & 262 & 5.4 \\
\hline Total TFs in Shewanella: & 213 & 76 & 77 & 26 & 51 & 33 & 280 & 144 & 48 & 169 & 818 & 66 & 131 & 97 & 381 & 282 & 134 & 32 & 17 & 16 & 16 & 16 & 16 & 16 & 16 & 16 & 14 & 27 & 3228 & - \\
\hline Average TF per genome: & 13 & 5 & 5 & 2 & 3 & 2 & 18 & 9 & 3 & 11 & 51 & 4 & 8 & 6 & 24 & 18 & 8 & 2 & 1 & 1 & 1 & 1 & 1 & 1 & 1 & 1 & 1 & - & 202 & - \\
\hline Overlap E. coli / Shewanella & 2 & 2 & 2 & 0 & 2 & 1 & 5 & 1 & 3 & 3 & 13 & 1 & 1 & 3 & 9 & 4 & 3 & 2 & 1 & 1 & 1 & 1 & 1 & 1 & 1 & 1 & 1 & 3 & 75 & - \\
\hline
\end{tabular}

Figure 2 Distribution by protein families of predicted DNA-binding transcription factors in the Shewanella genomes. 


\begin{tabular}{|c|c|c|c|c|c|c|c|c|c|c|c|c|c|c|c|c|c|c|c|}
\hline $\begin{array}{l}\text { TF } \\
\text { name }\end{array}$ & $\begin{array}{l}\text { S. one- } \\
\text { idensis } \\
\text { MR-1 }\end{array}$ & $\begin{array}{c}\text { S. } \\
\text { putrefa- } \\
\text { ciens } \\
\text { CN-32 }\end{array}$ & $\begin{array}{l}\text { S. putre- } \\
\text { faciens } \\
\text { W3-18-1 }\end{array}$ & $\begin{array}{c}\text { Shewa- } \\
\text { nella } \\
\text { sp. } \\
\text { ANA-3 }\end{array}$ & $\begin{array}{c}\text { Shewa- } \\
\text { nella } \\
\text { sp. MR- } \\
4 \\
\end{array}$ & $\begin{array}{c}\text { Shewa- } \\
\text { nella } \\
\text { sp. MR- } \\
7 \\
\end{array}$ & $\begin{array}{c}\text { S. } \\
\text { baltica } \\
\text { OS155 }\end{array}$ & $\begin{array}{c}\text { S. } \\
\text { denitri- } \\
\text { ficans } \\
\text { OS217 }\end{array}$ & $\begin{array}{l}\text { S. frigidi- } \\
\text { marina } \\
\text { NCIMB } \\
400\end{array}$ & $\begin{array}{c}\text { S. } \\
\text { amazo- } \\
\text { nensis } \\
\text { SB2B } \\
\end{array}$ & $\begin{array}{l}\text { S. } \\
\text { loihica } \\
\text { PV-4 }\end{array}$ & $\begin{array}{c}\text { S. } \\
\text { pealeana } \\
\text { ATCC } \\
700345\end{array}$ & $\begin{array}{c}\text { S.halifa- } \\
\text { xensis } \\
\text { HAW- } \\
\text { EB4 } \\
\end{array}$ & $\begin{array}{c}\text { S. } \\
\text { piezoto- } \\
\text { lerans } \\
\text { WP3 } \\
\end{array}$ & $\begin{array}{c}\text { S. } \\
\text { sediminis } \\
\text { HAW-EB3 }\end{array}$ & $\begin{array}{c}\text { S.woodyi } \\
\text { ATCCC } \\
51908\end{array}$ & $\begin{array}{l}\text { Pseudo- } \\
\text { monas } \\
\text { spp. }\end{array}$ & $\begin{array}{l}E \\
\text { coli }\end{array}$ & $\begin{array}{l}\text { Regulon } \\
\text { functional } \\
\text { role }\end{array}$ \\
\hline AgaR & - & - & - & + & + & + & - & - & - & + & - & - & - & - & - & - & - & rs & $\begin{array}{l}\text { GaINAc } \\
\text { utilization }\end{array}$ \\
\hline $\operatorname{ArgR}$ & + & + & + & + & + & + & + & + & + & + & + & + & + & + & + & + & - & rs & $\begin{array}{l}\text { Arginine } \\
\text { biosynthesis }\end{array}$ \\
\hline Betl & - & - & - & - & - & - & + & + & + & - & - & + & + & + & + & + & + & rs & $\begin{array}{l}\text { Osmotic } \\
\text { protection }\end{array}$ \\
\hline BirA & + & + & + & + & + & + & + & + & + & + & + & + & + & + & + & + & + & rs & $\begin{array}{l}\text { Biotin } \\
\text { biosynthesis }\end{array}$ \\
\hline Crp & $r$ & + & + & + & + & + & + & + & + & + & + & + & + & + & + & + & + & rs & $\begin{array}{l}\text { Global } \\
\text { regulon }\end{array}$ \\
\hline CueR & + & + & + & + & + & + & + & + & - & + & - & + & + & + & + & + & rs & rs & Copper efflux \\
\hline Dnr & - & - & - & - & - & - & - & + & - & + & + & - & - & - & - & - & rs & - & Denitrification \\
\hline FabR & + & + & + & + & + & + & + & + & + & + & + & + & + & + & + & + & + & rs & $\begin{array}{l}\text { Fatty acid } \\
\text { biosynthesis }\end{array}$ \\
\hline FadR & + & + & + & + & + & + & + & + & + & + & + & + & + & + & + & + & - & rs & $\begin{array}{l}\text { Fatty acid } \\
\text { degradation }\end{array}$ \\
\hline Fnr & $r$ & + & + & + & + & + & + & + & + & + & + & + & + & + & + & + & + & rs & $\begin{array}{l}\text { Global } \\
\text { regulon }\end{array}$ \\
\hline Fur & rs & + & + & + & + & + & + & + & + & + & + & + & + & + & + & + & rs & rs & $\begin{array}{l}\text { Iron } \\
\text { homeostasis }\end{array}$ \\
\hline GalR & - & - & - & - & - & - & - & - & - & - & - & - & - & - & - & + & - & rs & $\begin{array}{l}\text { Galactose } \\
\text { utilization }\end{array}$ \\
\hline GcvA & + & + & + & + & + & + & + & + & + & + & + & + & + & + & + & + & - & rs & $\begin{array}{l}\text { Glycine } \\
\text { metabolism }\end{array}$ \\
\hline GlmR & + & + & + & + & + & + & + & + & + & + & + & + & + & + & + & + & $r$ & - & LPS synthesis \\
\hline GntR & - & - & - & - & - & - & + & - & - & - & - & - & - & - & - & - & + & rs & $\begin{array}{l}\text { Gluconate } \\
\text { utilization }\end{array}$ \\
\hline HexR & + & + & + & + & + & + & + & + & + & + & + & + & + & + & + & + & rs & + & $\begin{array}{l}\text { Central sugar } \\
\text { metabolism }\end{array}$ \\
\hline HutC & + & + & + & + & + & + & + & + & + & + & + & + & + & + & + & + & $r$ & - & $\begin{array}{l}\text { Histidine } \\
\text { utilization }\end{array}$ \\
\hline$\| v Y$ & + & + & + & + & + & + & + & + & + & + & + & + & + & + & + & + & - & rs & $\begin{array}{l}\text { Isoleucine- } \\
\text { valine } \\
\text { synthesis }\end{array}$ \\
\hline$I s c R$ & + & + & + & + & + & + & + & + & + & + & + & + & + & + & + & + & + & rs & $\begin{array}{l}\text { Fe-S cluster } \\
\text { assembly }\end{array}$ \\
\hline LexA & + & + & + & + & + & + & + & + & + & + & + & + & + & + & + & + & rs & rs & $\begin{array}{l}\text { DNA damage } \\
\text { stress }\end{array}$ \\
\hline MetJ & + & + & + & + & + & + & + & + & + & + & + & + & + & + & + & + & - & rs & $\begin{array}{l}\text { Methionine } \\
\text { biosynthesis }\end{array}$ \\
\hline
\end{tabular}




\begin{tabular}{|c|c|c|c|c|c|c|c|c|c|c|c|c|c|c|c|c|c|c|c|}
\hline$\overline{\text { MetR }}$ & + & + & + & + & + & + & + & + & + & + & + & + & + & + & + & + & + & rs & $\begin{array}{l}\text { Methionine } \\
\text { biosynthesis }\end{array}$ \\
\hline ModE & + & - & - & + & + & + & + & - & - & - & - & - & - & - & - & - & + & rs & $\begin{array}{l}\text { Molybdenium } \\
\text { metabolism }\end{array}$ \\
\hline NanR & - & - & - & - & - & - & - & - & - & - & - & + & - & - & - & - & - & rs & $\begin{array}{l}\text { Sialic acid } \\
\text { utilization }\end{array}$ \\
\hline NarP & + & + & + & + & + & + & + & - & + & + & + & + & + & + & + & + & - & rs & $\begin{array}{l}\text { Nitrate/nitrite } \\
\text { respiration }\end{array}$ \\
\hline NhaR & + & + & + & + & + & + & + & + & + & + & + & + & + & + & + & + & - & rs & $\begin{array}{l}\text { Osmotic stress } \\
\text { protection }\end{array}$ \\
\hline NikR & - & - & - & - & - & - & - & - & - & - & - & + & + & - & + & - & + & rs & Nickel uptake \\
\hline NorR & - & + & + & + & + & + & - & - & + & + & + & + & + & + & + & + & + & rs & $\begin{array}{l}\text { Nitrosative } \\
\text { stress }\end{array}$ \\
\hline $\mathrm{NrdR}$ & + & + & + & + & + & + & + & + & + & + & + & + & + & + & + & + & + & rs & $\begin{array}{l}\text { Nucleotide } \\
\text { metabolism }\end{array}$ \\
\hline NrtR & rs & + & + & - & - & - & - & - & - & - & - & - & - & - & - & - & + & - & $\begin{array}{l}\text { Nicotinamide } \\
\text { utilization }\end{array}$ \\
\hline NsrR & + & + & + & + & + & + & + & + & + & + & + & + & + & + & + & + & - & rs & $\begin{array}{l}\text { Nitrosative } \\
\text { stress }\end{array}$ \\
\hline $\mathrm{NtrC}$ & + & + & + & + & + & + & + & + & + & + & + & + & + & + & + & + & rs & rs & $\begin{array}{l}\text { Nitrogen } \\
\text { assimilation }\end{array}$ \\
\hline PdhR & + & + & + & + & + & + & + & + & + & + & + & + & + & + & + & + & - & rs & $\begin{array}{l}\text { Pyruvate } \\
\text { metabolism }\end{array}$ \\
\hline PsrA & + & + & + & + & + & + & + & + & + & + & + & + & + & + & + & + & rs & - & $\begin{array}{l}\text { Fatty acid } \\
\text { degradation }\end{array}$ \\
\hline RbsR & - & - & - & - & - & - & - & - & - & - & - & + & + & - & - & - & + & rs & $\begin{array}{l}\text { Ribose } \\
\text { utilization }\end{array}$ \\
\hline SdaR & + & + & + & + & + & + & + & - & + & - & + & + & + & + & + & + & + & $r$ & $\begin{array}{l}\text { Glycerate } \\
\text { utilization }\end{array}$ \\
\hline SoxR & - & - & - & - & - & - & - & + & + & + & + & - & - & - & - & + & + & rs & $\begin{array}{l}\text { Superoxide } \\
\text { stress }\end{array}$ \\
\hline TorR & rs & - & - & + & + & + & + & - & + & + & + & + & + & + & + & + & - & rs & $\begin{array}{l}\text { TMAO } \\
\text { respiration }\end{array}$ \\
\hline TrpR & + & + & + & + & + & + & + & + & + & + & + & + & + & + & + & + & - & rs & $\begin{array}{l}\text { Amino acid } \\
\text { metabolism }\end{array}$ \\
\hline TyrR & + & + & + & + & + & + & + & + & + & + & + & + & + & + & + & + & $r s^{*}$ & rs & $\begin{array}{l}\text { Amino acid } \\
\text { metabolism }\end{array}$ \\
\hline ZntR & + & + & + & + & + & + & + & + & + & + & + & + & + & + & + & + & - & rs & Zinc efflux \\
\hline
\end{tabular}




\section{Table 2 Novel TF regulons predicted and reconstructed in Shewanella spp}

\begin{tabular}{|c|c|c|c|c|c|c|c|c|c|c|c|c|c|c|c|c|c|}
\hline $\begin{array}{l}\mathrm{TF} \\
\text { name }\end{array}$ & $\begin{array}{c}\text { S. } \\
\text { oneid- } \\
\text { ensis } \\
\text { MR-1 } \\
\end{array}$ & $\begin{array}{c}\text { S. } \\
\text { putrefa- } \\
\text { ciens } \\
\text { CN-32 } \\
\end{array}$ & $\begin{array}{c}\text { S.putrefa- } \\
\text { ciens W3- } \\
18-1\end{array}$ & $\begin{array}{l}\text { Shewa- } \\
\text { nella sp. } \\
\text { ANA-3 }\end{array}$ & $\begin{array}{l}\text { Shewa- } \\
\text { nella sp. } \\
\text { MR-4 }\end{array}$ & $\begin{array}{c}\text { Shewa- } \\
\text { nella sp. } \\
\text { MR-7 }\end{array}$ & $\begin{array}{c}\text { S. } \\
\text { baltica } \\
\text { OS155 }\end{array}$ & $\begin{array}{l}\text { S. denitri- } \\
\text { ficans } \\
\text { OS217 }\end{array}$ & $\begin{array}{l}\text { S. frigidi- } \\
\text { marina } \\
\text { NCIMB } 400\end{array}$ & $\begin{array}{c}\text { S. } \\
\text { amazo- } \\
\text { nensis } \\
\text { SB2B } \\
\end{array}$ & $\begin{array}{l}\text { S. } \\
\text { loihica } \\
\text { PV-4 }\end{array}$ & $\begin{array}{c}\text { S. } \\
\text { pealeana } \\
\text { ATCC } \\
700345 \\
\end{array}$ & $\begin{array}{c}\text { S.halifax- } \\
\text { ensis } \\
\text { HAW-EB4 }\end{array}$ & $\begin{array}{c}\text { S. } \\
\text { piezoto- } \\
\text { lerans } \\
\text { WP3 } \\
\end{array}$ & $\begin{array}{c}\text { S. } \\
\text { sediminis } \\
\text { HAW-EB3 }\end{array}$ & $\begin{array}{c}\text { S.woodyi } \\
\text { ATCCC } \\
51908\end{array}$ & $\begin{array}{l}\text { Regulon } \\
\text { functional role }\end{array}$ \\
\hline \multicolumn{18}{|c|}{ A. Regulons inferred from the analysis of metabolic pathways } \\
\hline AlgR $^{*}$ & - & - & - & - & - & - & - & - & + & - & - & - & - & - & - & - & $\begin{array}{l}\text { Hexuronate } \\
\text { utilization }\end{array}$ \\
\hline $\operatorname{AraR}^{*}$ & - & + & + & + & + & + & - & - & - & - & - & - & - & - & - & - & $\begin{array}{l}\text { Arabinose } \\
\text { utilization }\end{array}$ \\
\hline $\mathrm{Bg} \mathrm{R}^{*}$ & - & - & - & - & - & - & + & + & + & + & - & - & - & + & - & + & $\begin{array}{l}\text { Beta-glucoside } \\
\text { utilization }\end{array}$ \\
\hline $\mathrm{HmgR}^{*}$ & + & + & + & + & + & + & + & + & + & + & + & + & + & + & + & + & $\begin{array}{l}\text { Tyrosine } \\
\text { degradation }\end{array}$ \\
\hline HypR* & + & + & + & + & + & + & + & + & + & + & + & + & + & + & + & + & $\begin{array}{l}\text { Hydroxyproline } \\
\text { utilization }\end{array}$ \\
\hline LiuR & + & + & + & + & + & + & + & + & + & + & + & + & + & + & + & + & $\begin{array}{l}\text { Amino acid } \\
\text { utilization }\end{array}$ \\
\hline LIdR $^{*}$ & + & + & + & + & + & + & + & - & + & + & + & + & + & + & + & - & $\begin{array}{l}\text { Lactate } \\
\text { utilization }\end{array}$ \\
\hline$M a l R^{*}$ & + & - & - & + & + & + & + & + & + & + & + & - & - & + & - & + & $\begin{array}{l}\text { Maltodextrin } \\
\text { utilization }\end{array}$ \\
\hline ManR1* & - & - & - & - & - & + & - & - & - & + & - & - & - & - & - & - & $\begin{array}{l}\text { Mannose } \\
\text { utilization }\end{array}$ \\
\hline ManR2* & - & - & - & - & - & - & - & - & - & + & - & - & - & - & - & - & $\begin{array}{l}\text { Mannose } \\
\text { utilization }\end{array}$ \\
\hline MtIR2 & - & - & - & - & - & - & - & - & + & - & - & - & - & - & - & - & $\begin{array}{l}\text { Mannitol } \\
\text { utilization }\end{array}$ \\
\hline NagR & + & + & + & + & + & + & + & + & - & + & + & + & + & + & + & + & $\begin{array}{l}\text { GlcNAc } \\
\text { utilization }\end{array}$ \\
\hline $\mathrm{PflR}^{*}$ & - & + & + & - & - & - & - & - & + & + & + & + & + & + & + & + & $\begin{array}{l}\text { Formate } \\
\text { metabolism }\end{array}$ \\
\hline $\operatorname{PrpR}^{*}$ & + & + & + & + & + & + & + & + & + & + & + & + & + & + & + & + & $\begin{array}{l}\text { Propionate } \\
\text { utilization }\end{array}$ \\
\hline PUR* & + & + & + & + & + & + & + & + & + & + & + & + & + & + & + & + & $\begin{array}{l}\text { Purine } \\
\text { biosynthesis }\end{array}$ \\
\hline $\operatorname{ScrR}^{*}$ & - & - & - & + & + & + & + & - & + & - & - & - & - & - & - & - & $\begin{array}{l}\text { Sucrose } \\
\text { utilization }\end{array}$ \\
\hline TreR* & - & - & - & - & - & - & - & - & + & - & - & - & - & - & - & + & $\begin{array}{l}\text { Trehalose } \\
\text { utilization }\end{array}$ \\
\hline XItR* & - & - & - & - & - & - & - & - & - & - & - & + & + & - & - & - & Xylitol utilization \\
\hline
\end{tabular}


Table 2 Novel TF regulons predicted and reconstructed in Shewanella spp (Continued)

\begin{tabular}{|c|c|c|c|c|c|c|c|c|c|c|c|c|c|c|c|c|c|}
\hline \multicolumn{18}{|c|}{ B. Regulons inferred from the analysis of chromosomal gene clusters } \\
\hline $\mathrm{AzrR}^{*}$ & + & + & + & + & + & + & + & - & - & + & + & + & + & + & + & + & Superoxide stress \\
\hline CalR* & + & + & + & + & + & + & + & + & + & + & + & + & + & + & + & + & $\begin{array}{l}\text { Aromatics } \\
\text { utilization }\end{array}$ \\
\hline CueR2 & - & + & - & - & - & - & - & - & + & - & + & - & - & - & - & - & Copper efflux \\
\hline DeoR* & + & + & + & + & - & - & - & - & - & - & - & + & + & + & + & + & $\begin{array}{l}\text { Nucleoside } \\
\text { utilization }\end{array}$ \\
\hline PnuR* & + & + & + & - & - & - & + & - & - & - & - & - & - & - & - & - & NAD metabolism \\
\hline SO0072 & + & + & + & + & + & + & + & + & + & + & + & + & - & + & - & + & $\begin{array}{l}\text { ABC efflux } \\
\text { transporter }\end{array}$ \\
\hline SO0082 & + & - & - & - & + & - & + & - & + & - & - & - & - & - & - & - & $\begin{array}{l}\text { Benzoate } \\
\text { degradation }\end{array}$ \\
\hline SO0193 & + & + & + & + & + & + & + & - & + & + & + & - & - & - & - & - & $\begin{array}{l}\text { Phospholipid } \\
\text { synthesis }\end{array}$ \\
\hline SO0734 & + & + & + & - & - & - & + & - & - & - & - & - & - & - & - & - & $\begin{array}{l}\text { hypothetical } \\
\text { transporter }\end{array}$ \\
\hline SO1393 & + & - & - & + & + & + & - & - & - & - & + & - & - & - & - & - & hypothetical \\
\hline SO1415 & + & - & - & - & + & - & - & - & - & - & + & + & + & - & + & - & $\begin{array}{l}\text { flavocytochrome } \\
\text { c }\end{array}$ \\
\hline SO1578 & + & - & - & + & + & + & + & - & - & + & + & - & - & - & - & + & $\begin{array}{l}\text { Glutathione } \\
\text { detoxification }\end{array}$ \\
\hline SO1703 & + & + & + & + & + & + & + & + & - & + & + & + & + & + & + & - & multidrug efflux \\
\hline SO1758 & + & + & + & + & + & + & + & - & - & + & + & - & - & - & + & + & hypothetical \\
\hline SO2282 & + & + & + & + & + & + & + & - & - & + & - & + & + & + & - & + & $\begin{array}{l}\text { Amino acid } \\
\text { efflux }\end{array}$ \\
\hline SO3277 & + & + & + & + & + & + & + & + & + & + & + & + & + & + & + & + & multidrug efflux \\
\hline SO3385 & + & + & + & + & + & + & + & - & - & - & - & - & - & - & - & - & hypothetical \\
\hline SO3393 & + & + & + & + & + & + & + & + & + & + & + & - & - & + & + & + & $\begin{array}{l}\text { xenobiotic } \\
\text { reductase }\end{array}$ \\
\hline SO3494 & + & + & + & + & + & + & + & + & + & - & - & - & - & - & - & - & multidrug efflux \\
\hline SO3627 & + & - & - & - & - & - & - & - & - & - & - & - & - & - & + & - & $\begin{array}{l}\text { flavocytochrome } \\
\text { c }\end{array}$ \\
\hline SO4326 & + & - & - & + & - & - & - & - & - & + & - & + & + & + & + & + & multidrug efflux \\
\hline SO4468 & + & + & + & + & + & + & + & + & - & + & - & - & - & + & + & - & hypothetical \\
\hline SO4705 & + & + & + & + & + & + & + & + & + & + & + & + & + & + & + & + & hypothetical \\
\hline
\end{tabular}

The new TF names introduced in this work are marked by asterisks. 
known RNA regulatory elements (8 riboswitches and 6 transcriptional attenuators), is summarized in Additional file 4 and briefly described below. These data, in conjunction with the detailed information about DNA binding motifs and individual TFBSs, were compiled into the Shewanella collection of regulons that was uploaded to the RegPrecise database http://regprecise.lbl.gov[34].

\section{Reconstruction of regulons for previously characterized} regulators

Our general strategy of reconstructing regulons controlled by known TFs in a novel taxonomic group consists of the following steps: (i) search for orthologous TFs, (ii) collecting known target genes and TFBSs in a model genome, (iii) identifying orthologous target genes in the analyzed genomes and extracting their upstream regions, iv) application of a pattern recognition program, then constructing positional weight matrices (PWMs) and comparison of the newly identified TFBS motifs with the previously known sites/motifs in a model genome, v) search for additional sites in the analyzed genomes and consistency check or cross-species comparison of the predicted regulons (details are provided in Materials and Methods section; the strategy was also reviewed in [1]). For regulons with significantly different repertoire of target genes in the Shewanella spp., the above procedure was repeated starting at the third step in order to include novel candidate targets into the TFBS motif model and to revise the final gene content of the regulon.

For the Shewanella genomes, we performed regulon reconstruction for 41 TFs that are orthologous to previously characterized regulators (Table 1 ). The majority of these TFs have experimentally characterized orthologs in $\gamma$-proteobacteria from other lineages, such as $E$. coli (35 TFs) and/or Pseudomonas spp. (10 TFs), or had been previously studied in S. oneidensis (5 TFs) (Additional file 5). Among these regulators, there are 26 universal TFs, three strain-specific TFs and 13 TFs mosaically distributed in the Shewanella spp. The deduced TFBS motifs for 41 analyzed regulons in the Shewanella spp. were compared to previously known motifs for orthologous regulators in other $\gamma$-proteobacteria using the RegulonDB database for E. coli[35] and original publications for Pseudomonas spp. (Additional file 5). For three regulators with previously unknown binding sites (GlmR, HutC, and SdaR) we report, for the first time, the identity of their cognate TFBSs. The identified new motifs in Shewanella are conserved in upstream regions of known targets in $E$. coli (for SdaR) and Pseudomonas spp. (for GlmR and HutC) (data not shown). Two novel TFBS motifs (for AgaR and GcvA) in the Shewanella spp. are completely different from the respective motifs in E. coli. Five other TFBS motifs (for CueR, NhaR, PsrA, TrpR, and ZntR) in the Shewanella spp. are moderately different (3-4 mismatches in the conserved positions) from the known motifs of orthologous TFs previously described in E. coli and/or Pseudomonas spp. The remaining 31 Shewanella TFs appear to have binding motifs that are well conserved or only slightly different (1-2 mismatches in the conserved positions) from the motifs of their previously characterized orthologs.

\section{Inference of novel regulons for metabolic pathways and} chromosomal gene clusters

To identify novel regulons in the absence of experimental data, we used two types of potentially co-regulated gene sets: i) genes that constitute functional metabolic pathways (subsystems); and ii) genes derived from conserved gene neighborhoods that include a putative TF gene. To analyze metabolic subsystems and conserved chromosomal gene clusters projected across bacterial genomes we used the SEED database [36]. Each training set of potentially co-regulated operons was collected from 16 analyzed Shewanella genomes, and a collection of their upstream regions was used as an input for the motif-recognition program SignalX to predict a common DNA motif allowing a limited number of sequences to be ignored. At the next step, the Shewanella genomes were scanned with the constructed DNA motif to reveal the distribution of similar sites that were further verified by the consistency check procedure (reviewed in [1]). Finally, the genomic context of candidate co-regulated genes was used to attribute a potential TF to each novel regulon and associated DNA motif.

As a result, we inferred 41 novel regulons in Shewanella spp. including: i) 18 regulons for metabolic subsystems; and ii) 23 regulons for conserved chromosomal gene clusters (Table 2). The metabolic regulons from the first group control genes from the metabolic pathways of utilization of various carbohydrates, as well as formate, lactate, propionate, hydroxyproline/proline, tyrosine, and branched chain amino acids, and the purine biosynthesis pathway. All of these metabolic regulons except the purine regulon were assigned to a TF by a combination of different evidence types such as (i) positional clustering of target genes and TFs on the chromosome; ii) autoregulation of a TF by a cognate TFBS; iii) correlation in the phylogenetic pattern of co-occurrence of TFBSs and TFs in the genomes. Each of these novel TFs was functionally annotated in the SEED database (http://theseed.uchicago.edu) and tentatively named using an abbreviation of the target metabolic pathway/ genes. Hereinafter we mark the new names by asterisks.

Most of the novel metabolic TFs represent non-orthologous replacement of previously known TFs that control similar metabolic pathways in other lineages. For example, the propionate catabolism in the Enterobacteria is activated by the Fis-family regulator PrpR, 
whereas in the Shewanella spp. it is predicted to be controlled by a GntR-family TF PrpR*. The proline utilization is controlled by the Lrp-family activator PutR in the Vibrio spp. [37], the AraC-family activator PruR in the Pseudomonas spp. [38], and the predicted GntRfamily regulator HypR* in the Shewanella spp.. The homogentisate pathway of the tyrosine degradation is regulated by the IclR-type repressor HmgR in the Pseudomonas spp. [39], which is replaced by novel LysRfamily regulator HmgR* in the Shewanella spp.. Similar non-orthologous replacements of regulators have been detected for ten different carbohydrate catabolic pathways [33] and the lactate utilization system in the Shewanella spp. [30]. A novel purine-pathway regulon (named PUR*) with hitherto unknown cognate TF was inferred in Shewanella instead of PurR regulon previously characterized in other $\gamma$-proteobacteria including E. coli[40] and missing in the Shewanella spp.. Two novel regulators PflR* and XltR* were predicted to control metabolic pathways of pyruvate to formate fermentation and xylitol catabolism, whose regulation have not yet been previously described in any bacteria.

Functional annotations of novel TF regulons that were deduced from the analysis of conserved gene clusters are largely hypothetical and incomplete. Most of them are local regulators controlling one or two target operons (Additional file 4). Two novel TF regulators from the Crp family, named DeoR* and PnuR*, control candidate phosphorylases and transporters likely involved in the nucleoside/nicotinamide ribose utilization. A novel AsnC-type regulator AzrR* controls the azr-SO3586 operon, which encodes azoreductase and lactoylglutathione lyase that are likely involved in the superoxide stress protection. Novel regulator CalR* controls expression of the coniferyl aldehyde dehydrogenase calB that play a role in phytochemical aromatic compound utilization. Other inferred TF regulons appear to contain various hypothetical metabolite efflux transporters or flavocytochromes potentially involved in detoxification and undescribed respiratory processes, respectively.

\section{Identification of regulons for RNA regulatory elements}

We used known regulatory-RNA patterns from the Rfam database [41] to scan intergenic regions in 16 Shewanella genomes and analyzed the genomic context of candidate regulatory RNAs (Additional file 4).

Representatives of eight metabolite-responsive riboswitch families are scattered in most Shewanella genomes. The lysine, glycine, thiamine, cobalamin, riboflavin, and molybdenum cofactor riboswitches control genes for the respective amino acid / cofactor biosynthetic pathways and/or uptake transporters. The purine riboswitch controls adenosine deaminase and purine transporter. The riboswitch that binds second messenger cyclic di-GMP was found to control various subsets of genes in the Shewanella spp. including genes encoding extracellular proteins such as the chitin binding protein, chitinases, peptidases, and other hypothetical secreted proteins.

Six candidate attenuators that regulate operons responsible for the biosynthesis of branched chain amino acids, histidine, threonine, tryptophan, and phenylalanine in proteobacteria [42] are conserved in all analyzed Shewanella spp.

\section{Experimental validation of $\mathrm{N}$-acetylglucosamine- responsive regulon NagR in S. oneidensis MR-1}

A predicted transcriptional regulator NagR of the LacI family is a nonorthologous replacement of the NagC repressor from Enterobacteria. In addition to genes involved in Nag transport (nagP and $o m p^{\mathrm{Nag}}$ ) and biochemical conversion (nagK-nagB $\left.{ }^{\mathrm{II}}-n a g A\right)$, the reconstructed NagR regulon contains auxiliary components that are likely involved in chemotaxis and hydrolysis of chitin and/or chitooligosaccharides ( $m c p^{\mathrm{Nag}}-h e x$ and $c b p)$. Experimental validation of the reconstructed NagR regulon in S. oneidensis MR-1 was performed by both in vitro and in vivo approaches. The nagR gene was cloned and overexpressed in E. coli, and the recombinant protein was purified by $\mathrm{Ni}^{2+}$-chelating chromatography. We used electrophoretic mobility shift assay to test specific DNA-binding of the purified NagR protein to its predicted operator sites in upstream regions of the nagP (SO3503), nagK (SO3507), mcp ${ }^{\mathrm{Nag}}$ (SO3510), omp ${ }^{\mathrm{Nag}}$ (SO3514) and $c b p$ (SO1072) genes in S. oneidensis MR1. The maximal shift of the nagK DNA fragment observed at $100 \mathrm{nM}$ NagR was completely suppressed by the addition of $20 \mathrm{mM}$ of $\mathrm{N}$-acetylglucosamine, which was thus proven as a negative effector (Additional file 6A). Specific binding at $100 \mathrm{nM}$ NagR protein was also confirmed for the other four tested DNA fragments. To confirm the negative regulatory effect of NagR on gene expression in vivo, the S. oneidensis ?nagR targeted deletion mutant was constructed and relative transcript levels of the predicted NagR target genes were analyzed by quantitative RT-PCR. Relative mRNA levels of the $n a g P, n a g K, m c p^{\mathrm{Nag}}, o m p^{\mathrm{Nag}}$, and $c b p$ genes were elevated 15-, 50-, 16-11-, and 5-fold, respectively, in the ? $n a g R$ mutant compared to the wild-type strain when grown in the minimal medium supplied with lactate (Additional file 6B). These results confirm that NagR is a negative regulator of the chitin utilization genes that are de-repressed in response to $\mathrm{N}$-acetylglucosamine.

\section{Discussion}

\section{Conservation and variations in the regulatory network} evolution

Conservation of 5738 regulatory interactions identified for all predicted members of the reconstructed regulons 
across the Shewanella genus is shown in Additional file 4. Overall, the regulatory systems of the Shewanella spp. appears out to be considerably variable within the genus and quite distinct from other previously studied $\gamma$-proteobacteria. The observed variations can be classified in three distinct types: (i) "regulon expansion" in the Shewanella compared to other lineages that can be ranged from additions of several regulon members to largerscale shifts in the regulated metabolic pathways (e.g., HexR, PdhR, and TyrR regulons); (ii) "fuzzy regulons" when a regulon possess a conserved core and variable periphery within the Shewanella group (e.g., global regulons ArgR, Crp, Fur, NarP, and Fnr); (iii) "regulon loss or acquisition" when entire regulon (including all operons from a regulated pathway) is present only in some of the Shewanella species (e.g., for Dnr, ModE, BetI, and 17 regulons controlling various sugar utilization pathways [33]). Of course, this distinction is very schematic and in reality these types of behavior overlap. The mostly conserved regulatory interactions occur among TF regulons that are involved in the control of essential biosynthetic pathways (e.g., BirA, FabR, GlmR, IlvY, NrdR regulons), and universal stress responses (LexA and ZntR regulons).

To estimate the relative conservation of the predicted regulatory interactions in other lineages, we searched for orthologs of the putative regulon members in E.coli and compared the gene contents of the regulons reconstructed in the Shewanella and with orthologous regulons in E.coli captured in the RegulonDB database (Additional file 4). Similar analysis was performed for the Shewanella regulons characterized in the Pseudomonas spp. (but not in E. coli), including Dnr, GlmR, HexR, HutC, and PsrA (for references see Additional file 5). Among 468 cognate operons that belong to 42 studied regulons in the Shewanella spp., 138 operons (30\%) have orthologous known targets in E. coli or Pseudomonas, 223 operons ( $50 \%)$ lack orthologous operons, whereas the remaining 107 operons ( 20\%) have orthologous operons that are not under control of orthologous TFs in these species. Examples of impressive variations in the content of orthologous TF regulons in the Shewanella and E. coli are discussed below.

The comparison of the inferred regulons revealed striking differences in the strategies for regulation of the central carbohydrate and amino acid metabolism between the lineages comprising the Shewanella spp. and the Enterobacteria. In E. coli, two global regulators, FruR (fructose repressor/activator) and Crp (cAMPresponsive activator), control the central carbohydrate metabolism, whereas HexR (phospho-keto-deoxy-gluconate-responsive repressor) and PdhR (pyruvate repressor) are local regulators of glucose-6P dehydrogenase and pyruvate dehydrogenase, respectively. By contrast, the Shewanella spp. are predicted to use the HexR and PdhR regulators for the global control of the central carbohydrate metabolism and fermentation (Fig. 3). The FruR TF is absent in the Shewanella spp. that are not able to utilize fructose. The content and functional role of the Crp regulon is significantly different in the two lineages: the catabolism of carbohydrates and amino acids in the Enterobacteria, and the anaerobic respiration in the Shewanella spp. Most sugar catabolic pathways in the Shewanella spp. seem to be exclusively controlled by local sugar-responsive TFs that are often replaced by non-orthologous TFs (e.g., NagR vs. NagC for the Nacetylglucosamine utilization), and lack global co-regulation by Crp. Thus, the Shewanella spp. seem to lack many "feed-forward loops" that are characteristic for the regulation of sugar catabolism pathways in E.coli (when an operon is regulated by $\mathrm{Crp}$ and a local regulator that also is regulated by Crp) [43], thus may have a different strategy of sugar catabolism on mixed substrates.

Significant shifts in the regulon content were also identified for the TyrR, FadR, and FabR regulons (Fig. 3). In E. coli, the tyrosine- and phenylalanineresponsive regulator TyrR represses most aromatic amino acid biosynthetic enzymes and transporters encoded by multiple aro and tyr genes scattered on the chromosome, and activates the tyrosine transporter encoded by the $m t r$ gene. In the Shewanella spp., we identified TyrR as a master regulator of the degradation pathways for various amino acids, including phenylalanine (phhAB operon), tyrosine (fahA-maiA operon), branched chain amino acids $(l d h, b r n Q, l i u, i v d$, and $b k d$ operons), proline (putA gene), and oligopeptides (various peptidase genes), as well as some other pathways such as the glyoxylate shunt (aceBA operon), and the chorismate biosynthesis (aro $A$ gene). These findings are in accordance with the previously established role of PhhR, a TyrR ortholog in Pseudomonas spp., as an activator for phenylalanine and tyrosine degradation genes [44]. The fatty acid degradation pathway in the Shewanella app. and many other $\gamma$-proteobacteria is controlled by PsrA, whereas in the Enterobacteria the analogous pathway is regulated by FadR [4]. The Shewanella spp. also have a significantly reduced in size FadR regulon, which retains only two operons shared with the orthologous regulon of E. coli, fadIJ and fadL[4]. Finally, the fatty acid biosynthesis regulon FabR has only one gene, $f a b A$, which has conserved regulation in both $E$. coli and the Shewanella spp., whereas the remaining target genes were identified as a lineage-specific regulon extension.

\section{Interconnections between the predicted regulons in Shewanella spp}

The collection of the inferred Shewanella regulons contains at least 30 regulons (for 24 TFs and 6 regulatory 


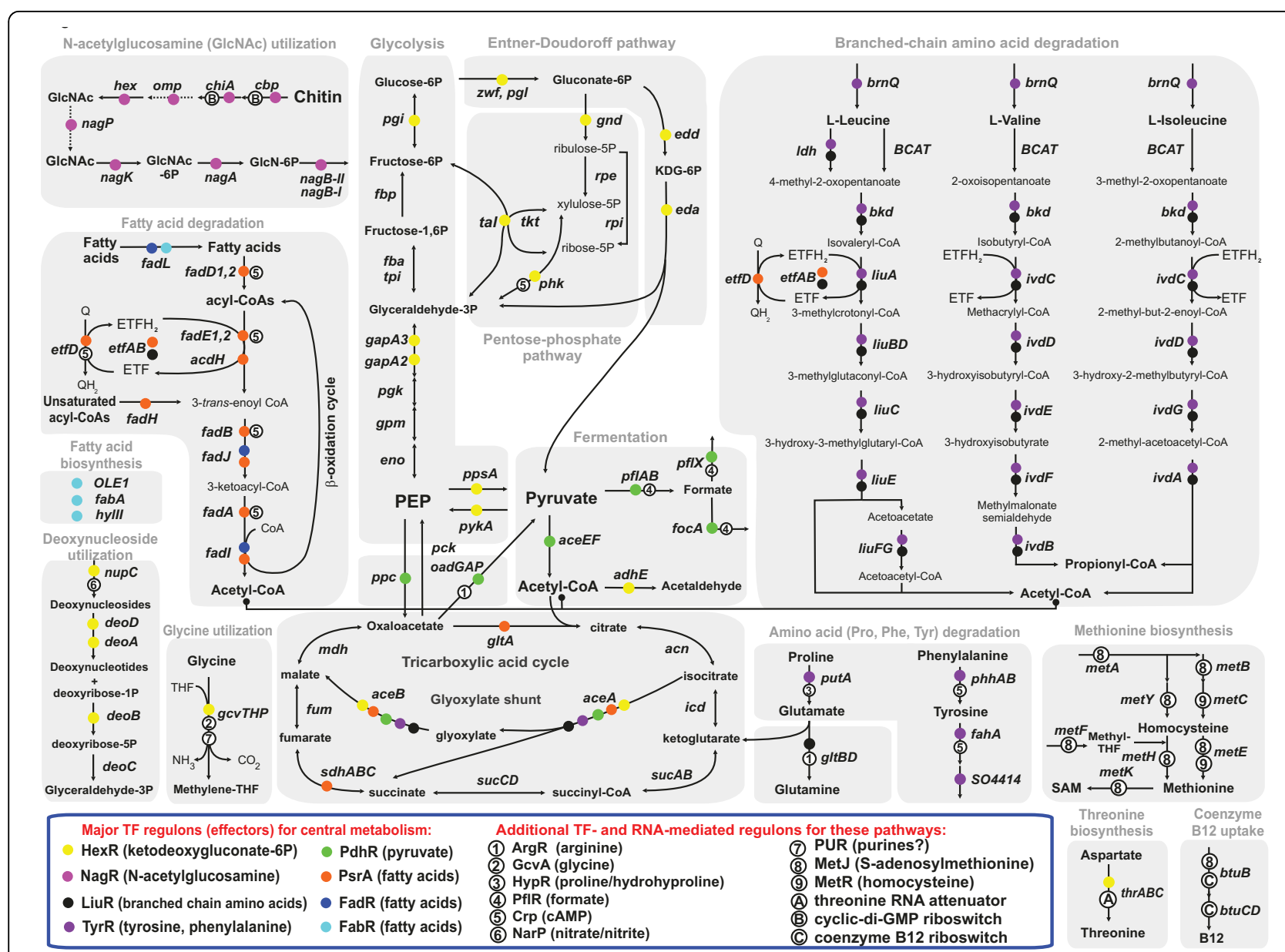

Figure 3 Metabolic context of the reconstructed regulons in Shewanella spp.

RNAs) that have at least one operon under simultaneous control of at least two regulators (Additional file 4). Most of the overlapping regulons control amino acid, fatty acid, nitrogen, and central carbohydrate metabolism (Fig. 3). The glyoxylate shunt operon aceBA controlled by five TFs is the most regulated operon in the current TRN model (see below). The glycine utilization operon $g c v T H P$ was found to be controlled by the glycine-responsive regulator GcvA, the central carbohydrate regulator HexR, and the novel purine biosynthesis regulator PUR*. In the predicted regulons, 14 operons are under overlapping control of three regulons, whereas $\sim 70$ operons are co-regulated by two regulons. At least four regulatory cascades between various TFs were identified in the Shewanella spp.: LiuR for tyrR, NarP for $c r p$, Crp for $h m g R$, and MetJ for metR, and only the latter cascade is conserved in $E$. coli.

The reconstructed TRN provides insight into interplay between several different TFs controlling multiple genes from the LiuR regulon (Fig. 4). LiuR is a MerR-family repressor that controls the branched chain amino acid
(Ile/Leu/Val) utilization in diverse proteobacteria [4]. In Shewanella spp., the predicted LiuR regulon was found to regulate Ile/Leu/Val operons (ldh, liu, $i v d$, and $b k d)$ and was expanded by additional members involved in the biosynthesis of glutamate $(g l t B D)$ and threonine $(\operatorname{thr} A B C)$, and the glyoxylate shunt $(a c e B A)$. Six out of nine LiuR-controlled operons are also regulated by the tyrosine/phenylalanine-responsive transcription factor TyrR [45]. Although TyrR in E. coli can act both as activator and repressor on its target genes, the mode of TyrR action on Shewanella targets is to be determined experimentally. Preliminary comparative analysis of relative positions of the TyrR- and LiuR-binding sites in Shewanella genomes (using multiple alignment of the promoter gene regions) suggests that TyrR probably acts as an activator for the $l d h, l i u, i v d$, and $b k d$ operons (data not shown). This supposition suggests that integrative effect of the LiuR and TyrR mediated control can be activation of their target genes in the simultaneous presence of Ile/Leu/Val and Tyr/Phe. Indeed, the expression data confirm strong up-regulation of the Ile/Leu/ 


\begin{tabular}{|c|c|c|c|c|c|c|c|c|c|}
\hline \multirow{2}{*}{$\begin{array}{l}\begin{array}{l}\text { Regulated } \\
\text { operon }\end{array} \\
\text { Idh }\end{array}$} & \multirow{2}{*}{$\begin{array}{l}\begin{array}{l}\text { Functional } \\
\text { role }\end{array} \\
\text { Leu/lle/Val utilization }\end{array}$} & \multicolumn{5}{|c|}{$\begin{array}{l}\text { Transcription } \\
\text { factors }\end{array}$} & $\begin{array}{l}\text { Change in gene } \\
\text { expression, } \\
\text { BU7_Cas condition }\end{array}$ & \multicolumn{2}{|c|}{$\begin{array}{l}\text { Gene expression } \\
\text { correlation matrix }\end{array}$} \\
\hline & & LiuR & TyrR & & & & & & \\
\hline ivdABCDEFG & Leu/lle/Val utilization & LiuR & TyrR & & & & & & \\
\hline liuRABCDEFG & Leu/lle/Val utilization & LiuR & TyrR & & & & & & \\
\hline bkdAB & Leu/lle/Val utilization & LiuR & TyrR & & & & & & \\
\hline etfBA & Leu/lle/Val, FA utilization & LiuR & & PsrA & & & & & \\
\hline tyrR & regulatory cascade & LiuR & TyrR & & & & & & \\
\hline glt $B D$ & Glu biosynthesis & LiuR & & & ArgR & & & & \\
\hline thrABC & Thr biosynthesis & LiuR & & & THR & & & & \\
\hline ace $B A$ & Glyoxylate shunt & LiuR & TyrR & PsrA & PdhR & HexR & & & \\
\hline Log2 (ratio) & & & & & & & Pearson $\mathrm{co}$ & tion coe & \\
\hline 2.00 & -2.00 & & & & & & 1.00 & 0.00 & -1.00 \\
\hline
\end{tabular}

Val utilization and glyoxylate shunt genes in the presence of casein-derived mixture of amino acids (Fig. 4).

In contrast, two amino acid biosynthetic operons are down-regulated in the same condition. This observation can be explained by additional regulatory mechanisms found for each of these operons. The glutamate synthase gltBD is also controlled by $\operatorname{ArgR}$, which is known to repress gene expression in the presence of arginine [46]. The threonine biosynthesis operon thrABC is also repressed by threonine availability using RNA attenuation mechanism [42].

Analysis of pairwise correlations for all LiuR-regulated genes based on $\sim 200$ microarray expression profiles available in the MicrobesOnLine database [47] allows us to identify two subregulons that have different gene expression patterns (Fig. 4). The first catabolic subregulon contains six operons, five of which are involved in the Ile/Leu/Val utilization, whereas the second subregulon has two biosynthetic operons and the glyoxylate shunt operon aceBA. The current TRN model has the largest number of regulatory interactions for the latter operon, which is controlled by five TFs including the Ile/Leu/Val repressor LiuR, the Tyr/Phe repressor/activator TyrR, the phospho-keto-deoxy-gluconate regulator HexR, the pyruvate repressor PdhR, and the fatty acid repressor PsrA. The glyoxylate shunt pathway plays a central metabolic role by providing intermediates required for amino acid biosynthesis, and being involved in the utilization of acetyl-CoA, a common product of the Ile/Leu/Val amino acids, fatty acids and carbohydrate degradation pathways [48].

\section{Conclusions and future perspectives}

By applying the comparative genomics approach, we tentatively defined the first reference collection of transcriptional regulons in 16 Shewanella genomes comprised of 82 orthologous groups of TFs, $~ 7,300 \mathrm{TF}-$ binding sites ( $\sim 40$ per genome), and 258 RNA regulatory motifs from 14 families. The resulting regulatory network contains $\sim 600$ regulated genes per genome that are mostly involved in the central metabolism, production of energy and biomass, metal ion homeostasis and stress responses. Although some diversity of the predicted regulons was observed within the Shewanella genus, the most significant diversification and adaptive evolution of TRNs were revealed by comparison with the established TRN in E. coli and related Enterobacteria. These differences are mostly attributed to: i) lineage specific regulon expansion and contraction for orthologous TFs that use conserved TFBS consensus motifs, and ii) involvement of non-orthologous TFs to control physiologically equivalent metabolic pathways in the two lineages of $\gamma$-proteobacteria.

The reconstructed regulons in S. oneidensis MR-1 are supported by available microarray expression data for the fur, crp, and etrA (fnr) knockout strains [25,26,28,29], as well as for the wild type strain grown on various carbon sources (inosine, $\mathrm{N}$-acetylglucosamine, amino acids, lactate, and pyruvate) [20]. Preliminary analysis of correlations in expression patterns of genes from predicted regulons was useful for the interpretation of the reconstructed TRN, as illustrated by the LiuR regulon example. We are currently expanding this approach to other data. Targeted experimental validation of eight novel regulons for central carbohydrate and amino acid metabolism in S. oneidensis MR-1 is currently underway. Previously we have characterized in vitro the novel NAD metabolism regulon NrtR [11] and in this work we present in vivo and in vitro validation of $\mathrm{N}$-acetylglucosamine utilization regulon NagR. Combined in vivo and in vitro experimental 
validation of the global carbohydrate metabolism regulon HexR and the assessment of its physiological role in Shewanella will be published elsewhere.

This work demonstrates the power of the comparative genomics approach in application to the reconstruction of transcriptional regulons in poorly studied groups of related bacteria. The reference set of the Shewanella regulons is the first taxonomy-wide collection of regulons obtained by this approach. It can be assessed in the RegPrecise database [34]. We anticipate a fast growth of taxonomy-wide regulon collections for other lineages in the near future. Regulatory interactions from the reconstructed regulons will provide an additional regulatory constrains for the recently published metabolic model of S. oneidensis MR-1 [22], allowing one to build an integrated model of metabolism and regulation. Such integrated model can be used for phenotype prediction, functional gene assignment and understanding of organism ecology. Finally, the reconstructed regulons were useful for the genome context-based prediction of novel functions of enzymes and transporters in previously uncharacterized carbohydrate utilization pathways in Shewanella spp. [33]

\section{Methods \\ Bioinformatics methods for regulon reconstruction and used databases}

The Shewanella spp. genomes were downloaded from the Genbank [49] (Fig. 1). The set of predicted DNAbinding TFs was extracted from the DBD database [50]. The locus_tag gene identifiers are used throughout. Orthologous proteins in 16 Shewanella genomes were defined in the previous work by the best bidirectional hits criterion [32]. Orthologous groups in Shewanella were named by either a common name of characterized protein, a novel name for proteins functionally annotated in this study, or by a locus_tag from S. oneidensis genome for uncharacterized proteins. Orthologs between proteins from different taxonomic groups (e.g. Shewanella and other $\gamma$-proteobacteria) were defined as bidirectional best hits with $30 \%$ of identity threshold using the Smith-Waterman algorithm implemented in the GenomeExplorer program [51]. In dubious cases orthologs were confirmed by construction of phylogenetic trees and comparative analysis of gene neighborhoods using the MicrobesOnline tree browse tool [47]. Functional gene assignments and metabolic subsystem analysis were performed using the SEED annotation/analysis tool http://theseed.uchicago.edu/FIG/index.cgi, which combines protein similarity search, positional gene clustering, and phylogenetic profiling of genes [36]. In addition, the InterPro [52], and PFAM [53] databases were used to verify protein functional and structural annotations.
For de novo identification of a candidate regulatory motif in the training set of potential upstream regions of genes (intergenic regions up to $350 \mathrm{bp}$ ) we used a simple iterative procedure DNA motif detection procedure implemented in the program SignalX [54]. Weak palindromes were selected in each region. Each palindrome was compared to all other palindromes, and the palindromes most similar to the initial one were used to make a profile. The candidate site score was defined as the sum of the respective positional nucleotide weights [7]. These profiles were used to scan the set of palindromes again, and the procedure was iterated until convergence. Thus a set of PWM profiles was constructed. A profile with largest information content was used as the recognition rule [55]. Each genome encoding the studied TF was scanned with the constructed motif profile using the GenomeExplorer software [51] and genes with candidate regulatory sites in the upstream regions were selected. The threshold for the site search was defined as the lowest score observed in the training set. Among new candidate members of a regulon, only genes having candidate sites conserved in at least two other genomes were retained for further analysis. We also included new candidate regulon members that are functionally related to the established regulon members. Additional and more detailed description of various scenario for regulon reconstruction using comparative genomics was reviewed in [1]. Analysis of large regulons (Fur, Crp, Fnr, NarP, LexA) was carried out using the web-based tool RegPredict allowing the comparative genomics-based regulon inference http://regpredict.lbl. gov[56]. The details of reconstructed regulons were captured and displayed in our recently developed database RegPrecise http://regprecise.lbl.gov[34]. For identification of RNA regulatory motif sequences we scanned complete genomes using tools and profiles available from the Rfam database [41]. Calculation of the Pearson coefficient for the LiuR-regulated genes was done by tools available at the MicrobesOnLine resource [47].

\section{Experimental methods for regulon validation}

The nagR (SO3516) gene cloned at a pET-derived vector containing the $\mathrm{T} 7$ promoter and $\mathrm{His}_{6}$ tag [57] was kindly provided by Frank Collart (Argonne National Laboratory, IL).

Protein purification. Recombinant proteins of nagR (SO3516) from S. oneidensis MR-1 was overexpressed as $\mathrm{N}$-terminal fusion with a $\mathrm{His}_{6}$ tag in E. coli strain BL21/ DE3. Cells were grown on $\mathrm{LB}$ media to $\mathrm{OD}_{600}=0.8$ at $37^{\circ} \mathrm{C}$, induced by $0.2 \mathrm{mM}$ IPTG, and harvested after $12 \mathrm{~h}$ shaking at $20^{\circ} \mathrm{C}$. Protein purification was performed using rapid Ni-NTA agarose minicolumn protocol as described [58]. Briefly, harvested cells were resuspended in $20 \mathrm{mM}$ HEPES buffer $\mathrm{pH} 7$ containing $100 \mathrm{mM}$ 
$\mathrm{NaCl}, 0.03 \%$ Brij 35, and $2 \mathrm{mM} \beta$-mercaptoethanol supplemented with $2 \mathrm{mM}$ phenylmethylsulfonyl fluoride and a protease inhibitor cocktail (Sigma-Aldrich). Lysozyme was added to $1 \mathrm{mg} / \mathrm{mL}$, and the cells were lyzed by freezing-thawing followed by sonication. After centrifugation at $18,000 \mathrm{rpm}$, the Tris- $\mathrm{HCl}$ buffer $(\mathrm{pH} \mathrm{8})$ was added to the supernatant (50 $\mathrm{mM}$, final concentration), and it was loaded onto a Ni-NTA agarose column (0.2 $\mathrm{ml})$. After washing with the starting buffer containing 1 $\mathrm{M} \mathrm{NaCl}$ and $0.3 \%$ Brij-35, bound proteins were eluted with $0.3 \mathrm{ml}$ of the starting buffer containing $250 \mathrm{mM}$ imidazole. Protein size, expression level, distribution between soluble and insoluble forms, and extent of purification were monitored by SDS-PAGE.

$\boldsymbol{q P C R}$. In-frame deletion mutagenesis of or $n a g R$ (SO3516) was performed using previously published method [33]. Genomic RNA was isolated from S. oneidensis MR-1 and $\triangle n a g R$ cells grown in minimal medium supplied with lactate and collected at O.D.(600) of 0.52 using the RNA purification kit from Promega (Madison, WI). Reverse transcription of total RNA was performed with random primers using iScript cDNA synthesis kit from BIO-RAD (Hercules, CA), following kits instructions. qPCR was performed using SYBR GreenER qPCR SupeMix Universal kit from Invitrogene (Carlsbad, CA). Transcript levels of the nagP (SO3503), nagK (SO3507), $m c p^{\mathrm{Nag}}$ (SO3510), omp ${ }^{\mathrm{Nag}}$ (SO3514), cbp (SO1072), SO0854, and $z w f$ (SO2489, used as a negative control) genes were measured and the results were normalized to the expression level of $16 \mathrm{~S}$ mRNA. Fold change was calculated by the $2^{-\Delta C T}$ method [59] as a ratio of normalized mRNA levels in $\Delta n a g R$ mutant and wild-type MR-1 strains.

DNA-binding assay. Interaction of purified recombinant protein NagR (SO3516) from S. oneidensis MR-1 with their cognate DNA motifs was assessed by EMSA technique using the following dsDNA segments obtained by PCR amplification or by custom synthesis of both complementary oligonucleotides (IDT, San Diego, CA), annealing and purification. One of the primers was 5'-biotinylated (IDT). By using S. oneidensis MR-1 DNA as the template, we amplified DNA fragments from the following upstream gene regions: SO1072 (89 bp), SO3507 (69 bp), SO3510 (64 bp), SO3514 (69 bp), SO3503 (62 bp), SO0854 (67 bp). For EMSA, the biotin-labeled DNA (0.1 or $1 \mathrm{nM})$ was incubated with the increasing amount of purified NagR (0$100 \mathrm{nM}$ ) in a total volume of $20 \mu \mathrm{l}$. The binding buffer contains Tris- $\mathrm{HCl} 20 \mathrm{mM}, \mathrm{KCl} 150 \mathrm{mM}, \mathrm{MgCl} 25 \mathrm{mM}$, DTT 1mM, EDTA 1mM, 0.05\% NP-40, 2.5\% glycerol. The poly(dI-dC) (Sigma) was added as nonspecific competitor DNA at $\sim 10^{4}$-fold molar excess over labeled target DNA to reduce nonspecific binding. After $25 \mathrm{~min}$ incubation at room temperature, the reaction mixtures were separated by electrophoresis on a $5 \%$ native polyacrylamide gel in $0.5 \times$ Tris-borate-EDTA for $90 \mathrm{~min}$ at $90 \mathrm{~V}$, at $4^{\circ} \mathrm{C}$. The gel was transferred by electrophoresis (30 min, at $380 \mathrm{~mA}$ ) onto a nylon membrane (Pierce, Rockford, Ill.) and fixed by UV cross-linking. Biotin-labeled DNA was detected with the LightShift Chemiluminescent EMSA kit (Pierce, Rockford, Ill.), as recommended by the manufacturer. The effect of $N$-acetyl-glucosamine on NagR-DNA binding was tested by addition of $20 \mathrm{mM}$ of $N$-acetylglucosamine to the incubation mixture.

\section{Additional material}

Additional file 1: Rodionov_AF1.xls - Repertoire of DNA-binding transcription factors identified in 16 genomes of Shewanella spp.

Additional file 2: Rodionov_AF2.xls - Transcription factors universally conserved in Shewanella spp.

Additional file 3: Rodionov_AF3.xls - Functional classifiaction of 73 transcription factors conserved between $E$. coli and 16 Shewanella strains.

Additional file 4: Rodionov_AF4.xls - Functional content and conservation of reconstructed TF and RNA regulons in Shewanella genomes.

Additional file 5: Rodionov_AF5.pdf - Comparison of predicted TFBS motifs in Shewanella spp. to the previously characterized orthologous regulators in model species.

Additional file 6: Rodionov_AF6.pdf - Experimental validation of NagR regulon in S. oneidensis MR-1. (A) Electrophoretic mobility shift assays to assess NagR protein binding to their predicted DNA operators; (B) Differential regulation of NagR controlled genes determined by quantitative qPCR.

List of abbreviations used

TF: transcription factor; TFBS: transcription factor-binding site; TRN: transcriptional regulatory network.

\section{Acknowledgments}

This research was supported by the Office of Science, Office of Biological and Environmental Research, of the U.S. Department of Energy under Contracts DE-AC02-05CH11231 with Lawrence Berkeley National Laboratory (ENIGMA SFA), DE-AC05-76RLO with Pacific Northwest National Laboratory (SBR FSFA); and DE-SC0004999 with Sanford-Burnham Medical Research Institute and Lawrence Berkeley National Laboratory. Additional funding was provided by National Science Foundation (DBI-0850546 to D.A.R. and R.O.); Russian Foundation for Basic Research (08-04-01000 to A.E.K., 09-04-92745 and 10-04-00431 to M.S.G., 10-04-01768 to D.A.R., E.D.S. by 09-04-92742), Russian Academy of Sciences (program 'Molecular and Cellular Biology' to D. A.R and M.S.G.); Russian Agency on Education (P2581 to E.D.S.); Russian Science Agency (2.740.11.0101 to M.S.G.).

This article has been published as part of BMC Genomics Volume 12 Supplement 1, 2011: Validation methods for functional genome annotation. The full contents of the supplement are available online at http://www. biomedcentral.com/1471-2164/12?issue=S1

\section{Author details}

'Sanford-Burnham Medical Research Institute, La Jolla, California, USA ${ }^{2}$ Institute for Information Transmission Problems, Russian Academy of Sciences, Moscow, Russia. ${ }^{3}$ Lawrence Berkeley National Laboratory, Berkeley, California, USA. ${ }^{4}$ Faculty of Bioengineering and Bioinformatics, Moscow State University, Moscow, Russia. ${ }^{5}$ State Scientific Center GosNIIGenetika, Moscow, Russia. ${ }^{6}$ Fellowship for Interpretation of Genomes, Burr Ridge, Illinois, USA. 
${ }^{7}$ Biological Sciences Division, Pacific Northwest National Laboratory, Richland, Washington, USA. ${ }^{8}$ Department of Energy Joint Genome Institute, Walnut Creek, California, USA.

\section{Authors' contributions}

DARO, ALO and MSG conceived and supervised the research, and wrote the manuscript. DARo, IAR, DARa, AEK, EAP, AVG, ONL, GYK performed comparative genomic analysis to infer novel transcription factor regulons. MDK identified riboswitches. PSN developed RegPredict tool and RegPrecise database, performed propagation of regulons. DARo and PSN performed correlation analysis using expression data. EDS and RO performed computational similarity searches and gene annotation in the SEED database. MFR produced manually annotated table of orthologs and provided targeted gene knockout strains in Shewanella. XL carried out validation experiments for NagR regulon. JKF, ID and APA contributed to the development of the manuscript and design of the study. All authors read and approved the final manuscript.

\section{Competing interests}

The authors declare that they have no competing interests.

Published: 15 June 2011

\section{References}

1. Rodionov DA: Comparative genomic reconstruction of transcriptional regulatory networks in bacteria. Chem Rev 2007, 107:3467-3497.

2. Minchin SD, Busby SJ: Analysis of mechanisms of activation and repression at bacterial promoters. Methods 2009, 47:6-12.

3. Grainger DC, Lee DJ, Busby SJ: Direct methods for studying transcription regulatory proteins and RNA polymerase in bacteria. Curr Opin Microbiol 2009, 12:531-535.

4. Kazakov AE, Rodionov DA, Alm E, Arkin AP, Dubchak I, Gelfand MS: Comparative genomics of regulation of fatty acid and branched-chain amino acid utilization in proteobacteria. J Bacteriol 2009, 191:52-64.

5. Laikova ON, Mironov AA, Gelfand MS: Computational analysis of the transcriptional regulation of pentose utilization systems in the gamma subdivision of Proteobacteria. FEMS Microbiol Lett 2001, 205:315-322.

6. Makarova KS, Mironov AA, Gelfand MS: Conservation of the binding site for the arginine repressor in all bacterial lineages. Genome Biol 2001, 2: RESEARCH0013.

7. Mironov AA, Koonin EV, Roytberg MA, Gelfand MS: Computer analysis of transcription regulatory patterns in completely sequenced bacterial genomes. Nucleic Acids Res 1999, 27:2981-2989.

8. Panina EM, Mironov AA, Gelfand MS: Comparative analysis of FUR regulons in gamma-proteobacteria. Nucleic Acids Res 2001, 29:5195-5206.

9. Permina EA, Kazakov AE, Kalinina OV, Gelfand MS: Comparative genomics of regulation of heavy metal resistance in Eubacteria. BMC Microbiol 2006, 6:49.

10. Ravcheev DA, Gerasimova AV, Mironov AA, Gelfand MS: Comparative genomic analysis of regulation of anaerobic respiration in ten genomes from three families of gamma-proteobacteria (Enterobacteriaceae, Pasteurellaceae, Vibrionaceae). BMC Genomics 2007, 8:54.

11. Rodionov DA, De Ingeniis J, Mancini C, Cimadamore F, Zhang $H$, Osterman AL, Raffaelli N: Transcriptional regulation of NAD metabolism in bacteria: NrtR family of Nudix-related regulators. Nucleic Acids Res 2008, 36:2047-2059.

12. Rodionov DA, Dubchak IL, Arkin AP, Alm EJ, Gelfand MS: Dissimilatory metabolism of nitrogen oxides in bacteria: comparative reconstruction of transcriptional networks. PLoS Comput Biol 2005, 1:e55.

13. Rodionov DA, Gelfand MS: Identification of a bacterial regulatory system for ribonucleotide reductases by phylogenetic profiling. Trends Genet 2005, 21:385-389.

14. Rodionov DA, Mironov AA, Gelfand MS: Conservation of the biotin regulon and the BirA regulatory signal in Eubacteria and Archaea. Genome Res 2002, 12:1507-1516.

15. Rodionov DA, Mironov AA, Rakhmaninova AB, Gelfand MS: Transcriptional regulation of transport and utilization systems for hexuronides, hexuronates and hexonates in gamma purple bacteria. Mol Microbiol 2000, 38:673-683.

16. Faith JJ, Hayete B, Thaden JT, Mogno I, Wierzbowski J, Cottarel G, Kasif S, Collins JJ, Gardner TS: Large-scale mapping and validation of Escherichia coli transcriptional regulation from a compendium of expression profiles. PLOS Biol 2007, 5:e8.

17. Liu J, Xu X, Stormo GD: The cis-regulatory map of Shewanella genomes. Nucleic Acids Res 2008, 36:5376-5390.

18. Fredrickson JK, Romine MF, Beliaev AS, Auchtung JM, Driscoll ME, Gardner TS, Nealson KH, Osterman AL, Pinchuk G, Reed JL, et al: Towards environmental systems biology of Shewanella. Nat Rev Microbiol 2008, 6:592-603.

19. Hau HH, Gralnick JA: Ecology and biotechnology of the genus Shewanella. Annu Rev Microbiol 2007, 61:237-258.

20. Driscoll ME, Romine MF, Juhn FS, Serres MH, McCue LA, Beliaev AS, Fredrickson JK, Gardner TS: Identification of diverse carbon utilization pathways in Shewanella oneidensis MR-1 via expression profiling. Genome Inform 2007, 18:287-298.

21. Gupta N, Tanner S, Jaitly N, Adkins JN, Lipton M, Edwards R, Romine M, Osterman A, Bafna V, Smith RD, Pevzner PA: Whole proteome analysis of post-translational modifications: applications of mass-spectrometry for proteogenomic annotation. Genome Res 2007, 17:1362-1377.

22. Pinchuk GE, Hill EA, Geydebrekht OV, De Ingeniis J, Zhang X, Osterman AL, Scott JH, Reed JL, Romine MF, Konopka AE, et al: Constraint-based model of Shewanella oneidensis MR-1 metabolism: a tool for data analysis and hypothesis generation. PLoS Comput Biol 2010, 6:e1000822.

23. Gralnick JA, Brown CT, Newman DK: Anaerobic regulation by an atypical Arc system in Shewanella oneidensis. Mol Microbiol 2005, 56:1347-1357.

24. Gao H, Wang X, Yang ZK, Palzkill T, Zhou J: Probing regulon of ArcA in Shewanella oneidensis MR-1 by integrated genomic analyses. BMC Genomics 2008, 9:42.

25. Saffarini DA, Schultz R, Beliaev A: Involvement of cyclic AMP (cAMP) and CAMP receptor protein in anaerobic respiration of Shewanella oneidensis. J Bacteriol 2003, 185:3668-3671.

26. Beliaev AS, Thompson DK, Fields MW, Wu L, Lies DP, Nealson KH, Zhou J: Microarray transcription profiling of a Shewanella oneidensis etrA mutant. J Bacteriol 2002, 184:4612-4616.

27. Bordi C, Ansaldi M, Gon S, Jourlin-Castelli C, lobbi-Nivol C, Mejean V: Genes regulated by TorR, the trimethylamine oxide response regulator of Shewanella oneidensis. J Bacteriol 2004, 186:4502-4509.

28. Wan XF, Verberkmoes NC, McCue LA, Stanek D, Connelly H, Hauser LJ, Wu L, Liu X, Yan T, Leaphart A, et al: Transcriptomic and proteomic characterization of the Fur modulon in the metal-reducing bacterium Shewanella oneidensis. J Bacteriol 2004, 186:8385-8400.

29. Yang Y, Harris DP, Luo F, Wu L, Parsons AB, Palumbo AV, Zhou J: Characterization of the Shewanella oneidensis Fur gene: roles in iron and acid tolerance response. BMC Genomics 2008, 9(Suppl 1):S11.

30. Pinchuk GE, Rodionov DA, Yang C, Li X, Osterman AL, Dervyn E, Geydebrekht OV, Reed SB, Romine MF, Collart FR, et al: Genomic reconstruction of Shewanella oneidensis MR-1 metabolism reveals a previously uncharacterized machinery for lactate utilization. Proc Natl Acad Sci U S A 2009, 106:2874-2879.

31. Yang C, Rodionov DA, Li X, Laikova ON, Gelfand MS, Zagnitko OP, Romine MF, Obraztsova AY, Nealson KH, Osterman AL: Comparative genomics and experimental characterization of $\mathrm{N}$-acetylglucosamine utilization pathway of Shewanella oneidensis. J Biol Chem 2006, 281:29872-29885.

32. Konstantinidis KT, Serres MH, Romine MF, Rodrigues JL, Auchtung J, McCue LA, Lipton MS, Obraztsova A, Giometti CS, Nealson KH, et al: Comparative systems biology across an evolutionary gradient within the Shewanella genus. Proc Natl Acad Sci U S A 2009, 106:15909-15914.

33. Rodionov DA, Yang C, Li X, Rodionova IA, Wang Y, Obraztsova AY, Zagnitko O, Overbeek R, Romine MF, Reed S, et al: Genomic encyclopedia of sugar utilization pathways in the Shewanella genus. BMC Genomics 2010, 11:494.

34. Novichkov PS, Laikova ON, Novichkova ES, Gelfand MS, Arkin AP, Dubchak I, Rodionov DA: RegPrecise: a database of curated genomic inferences of transcriptional regulatory interactions in prokaryotes. Nucleic Acids Res 2010, 38:D111-118.

35. Gama-Castro S, Jimenez-Jacinto V, Peralta-Gil M, Santos-Zavaleta A, Penaloza-Spinola MI, Contreras-Moreira B, Segura-Salazar J, Muniz-Rascado L, Martinez-Flores I, Salgado H, et al: RegulonDB (version 6.0): gene regulation model of Escherichia coli K-12 beyond transcription, active (experimental) annotated promoters and Textpresso navigation. Nucleic Acids Res 2008, 36:D120-124. 
36. Overbeek R, Begley T, Butler RM, Choudhuri JV, Chuang HY, Cohoon M, de Crecy-Lagard V, Diaz N, Disz T, Edwards R, et al: The subsystems approach to genome annotation and its use in the project to annotate 1000 genomes. Nucleic Acids Res 2005, 33:5691-5702.

37. Lee $\mathrm{H}$, Choi $\mathrm{SH}$ : Coactivation of Vibrio vulnificus putAP operon by cAMP receptor protein and PutR through cooperative binding to overlapping sites. Mol Microbiol 2006, 60:513-524.

38. Nakada Y, Nishijyo T, Itoh Y: Divergent structure and regulatory mechanism of proline catabolic systems: characterization of the putAP proline catabolic operon of Pseudomonas aeruginosa PAO1 and its regulation by PruR, an AraC/XylS family protein. J Bacteriol 2002, 184:5633-5640

39. Arias-Barrau E, Olivera ER, Luengo JM, Fernandez C, Galan B, Garcia JL, Diaz E, Minambres B: The homogentisate pathway: a central catabolic pathway involved in the degradation of L-phenylalanine, L-tyrosine, and 3-hydroxyphenylacetate in Pseudomonas putida. J Bacteriol 2004, 186:5062-5077.

40. Ravcheev DA, Gel'fand MS, Mironov AA, Rakhmaninova AB: [Purine regulon of gamma-proteobacteria: a detailed description]. Genetika 2002, 38:1203-1214.

41. Gardner PP, Daub J, Tate JG, Nawrocki EP, Kolbe DL, Lindgreen S, Wilkinson AC, Finn RD, Griffiths-Jones S, Eddy SR, Bateman A: Rfam: updates to the RNA families database. Nucleic Acids Res 2009, 37: D136-140.

42. Vitreschak AG, Lyubetskaya EV, Shirshin MA, Gelfand MS, Lyubetsky VA: Attenuation regulation of amino acid biosynthetic operons in proteobacteria: comparative genomics analysis. FEMS Microbiol Lett 2004, 234:357-370

43. Gonzalez Perez AD, Gonzalez Gonzalez E, Espinosa Angarica V, Vasconcelos AT, Collado-Vides J: Impact of Transcription Units rearrangement on the evolution of the regulatory network of gammaproteobacteria. BMC Genomics 2008, 9:128.

44. Panina EM, Vitreschak AG, Mironov AA, Gelfand MS: Regulation of aromatic amino acid biosynthesis in gamma-proteobacteria. J Mol Microbiol Biotechnol 2001, 3:529-543.

45. Palmer GC, Palmer KL, Jorth PA, Whiteley M: Characterization of the Pseudomonas aeruginosa transcriptional response to phenylalanine and tyrosine. J Bacteriol 2010, 192:2722-2728.

46. Paul L, Mishra PK, Blumenthal RM, Matthews RG: Integration of regulatory signals through involvement of multiple global regulators: control of the Escherichia coli gltBDF operon by Lrp, IHF, Crp, and ArgR. BMC Microbiol 2007, 7:2.

47. Dehal PS, Joachimiak MP, Price MN, Bates JT, Baumohl JK, Chivian D, Friedland GD, Huang KH, Keller K, Novichkov PS, et al: MicrobesOnline: an integrated portal for comparative and functional genomics. Nucleic Acids Res 2010, 38:D396-400.

48. Cozzone AJ: Regulation of acetate metabolism by protein phosphorylation in enteric bacteria. Annu Rev Microbiol 1998, 52:127-164.

49. Benson DA, Karsch-Mizrachi I, Lipman DJ, Ostell J, Sayers EW: GenBank. Nucleic Acids Res 38:D46-D51.

50. Wilson D, Charoensawan V, Kummerfeld SK, Teichmann SA: DBDtaxonomically broad transcription factor predictions: new content and functionality. Nucleic Acids Res 2008, 36:D88-92.

51. Mironov AA, Vinokurova NP, Gelfand MS: GenomeExplorer: software for analysis of complete bacterial genomes. Mol Bio I(Mosk) 2000, 34:222-231.

52. Hunter S, Apweiler R, Attwood TK, Bairoch A, Bateman A, Binns D, Bork P, Das $U$, Daugherty $L$, Duquenne $L$, et al: InterPro: the integrative protein signature database. Nucleic Acids Res 2009, 37:D211-215.

53. Finn RD, Mistry J, Tate J, Coggill P, Heger A, Pollington JE, Gavin OL, Gunasekaran P, Ceric G, Forslund K, et al: The Pfam protein families database. Nucleic Acids Res 2010, 38:D211-222

54. Gelfand MS, Koonin EV, Mironov AA: Prediction of transcription regulatory sites in Archaea by a comparative genomic approach. Nucleic Acids Res 2000, 28:695-705.

55. Schneider TD, Stormo GD, Gold L, A E: Information content of binding sites on nucleotide sequences. J Mol Biol 1986, 188:415-431.

56. Novichkov PS, Rodionov DA, Stavrovskaya ED, Novichkova ES, Kazakov AE Gelfand MS, Arkin AP, Mironov AA, Dubchak I: RegPredict: an integrated system for regulon inference in prokaryotes by comparative genomics approach. Nucleic Acids Res 2010, 38:W299-307.
57. Lin CT, Moore PA, Auberry DL, Landorf EV, Peppler T, Victry KD, Collart FR, Kery $\mathrm{V}$ : Automated purification of recombinant proteins: combining high-throughput with high yield. Protein Expr Purif 2006, 47:16-24.

58. Osterman AL, Lueder DV, Quick M, Myers D, Canagarajah BJ, Phillips MA Domain organization and a protease-sensitive loop in eukaryotic ornithine decarboxylase. Biochemistry 1995, 34:13431-13436.

59. Livak KJ, Schmittgen TD: Analysis of relative gene expression data using real-time quantitative PCR and the 2(-Delta Delta C(T)) Method. Methods 2001, 25:402-408.

doi:10.1186/1471-2164-12-S1-S3

Cite this article as: Rodionov et al: Comparative genomic reconstruction of transcriptional networks controlling central metabolism in the Shewanella genus. BMC Genomics 2011 12(Suppl 1):S3.

\section{Submit your next manuscript to BioMed Central and take full advantage of:}

- Convenient online submission

- Thorough peer review

- No space constraints or color figure charges

- Immediate publication on acceptance

- Inclusion in PubMed, CAS, Scopus and Google Scholar

- Research which is freely available for redistribution

Submit your manuscript at www.biomedcentral.com/submit
C) Biomed Central 